\title{
Níveis de sódio em dietas para codorna japonesa em pico de postura
}

\section{Sergio Luiz de Toledo Barreto ${ }^{1}$, Marcelle Santana de Araujoㄹ, Regina Tie Umigi², Weylisson César Oliveira Moura ${ }^{3}$, Carlos Henrique Rocha Costa ${ }^{2}$, Mariele Freitas Sousa ${ }^{4}$}

\author{
${ }^{1}$ Depto. de Zootecnia da UFV - Av. P.H. Holfs, s/n - CEP: 36570-000 - Viçosa-MG. \\ 2 Doutorando do Depto. de Zootecnia da UFV. \\ 3 Mestrando do Depto. de Zootecnia da UFV. \\ ${ }^{4}$ Graduanda do Depto. de Zootecnia da UFV.
}

RESUMO - Um experimento foi realizado objetivando-se verificar o efeito de diferentes níveis de sódio (Na) na dieta sobre o desempenho e a qualidade dos ovos de codornas japonesas durante o pico de postura (69 a 132 dias de idade). Foram utilizadas 300 codornas japonesas com 69 dias de idade, distribuídas em 30 unidades experimentais, cada uma com dez aves. O delineamento foi inteiramente casualizado com cinco tratamentos e seis repetições. Os níveis de Na estudados foram 0,017; 0,$083 ; 0,149 ; 0,215 ; 0,281 \%$ e corresponderam a 0,$000 ; 0,166 ; 0,332 ; 0,499 ; 0,665 \%$, respectivamente, de inclusão de sal comum nas dietas. As dietas experimentais foram isoprotéicas (20,0\% de PB), isocalóricas (2.900 kcal de EM/kg) e isocálcicas (3,2\% de Ca). Verificou-se melhora linear na produção de ovos, na conversão alimentar por massa de ovos, nas porcentagens de albúmem e de gema no ovo e na gravidade específica dos ovos com o aumento do nível de Na na dieta. Houve comportamento quadrático para o consumo de ração, o peso médio do ovo, a massa de ovo/ave/dia, o peso corporal final, a espessura de casca e a unidade Haugh do ovo. Não foram encontrados efeitos dos níveis de Na sobre a conversão alimentar por dúzia de ovos, a porcentagem de casca no ovo, a viabilidade e a umidade na excreta das aves. Dietas contendo 0,281\% de Na, correspondendo a um consumo diário de 69,1 mg de Na/ave, melhoram o desempenho e a qualidade do ovo de codornas japonesas durante o pico de produção. No entanto, desempenho satisfatório, com base na produção de ovos/ave/dia e de ovos comercializáveis pode ser obtido com aves alimentadas com ração contendo 0,149\% de Na.

Palavras-chave: Coturnix coturnix japonica, produção de ovos, qualidade de ovos, sal comum

\section{Sodium levels in laying Japanese quail diet on the peak production}

\begin{abstract}
An experiment was carried out to verify the effect of different dietary Na levels on the performance and egg quality of laying Japanese quail during the peak of production (69 to 132 days old). A total of 300 laying Japanese quails aged 69 days was allocated to 30 experimental units with ten birds each. The quails were assigned to a completely randomized design, with five treatments, six replicates. The Na levels studied were $0.017,0.083,0.149,0.215$, and $0.281 \%$ corresponding to $0.000,0.166,0.332,0.499$, and $0.665 \%$, respectively, of salt inclusion in the diets. The experimental diets were isoproteic $(20.0 \% \mathrm{CP})$, isoenergetic $(2,900 \mathrm{kcal} \mathrm{ME} / \mathrm{kg})$ and isocalcium $(3.2 \% \mathrm{Ca})$. It was observed linear increase of egg production, feed conversion by egg mass, albumen and yolk percentage and egg specific gravity with the increase of Na in the diet. Quadratic effect was observed for feed intake, weight egg, egg mass/bird.day, final body weight, eggshell thickness and Haugh unit. No effect of Na levels was observed of on feed conversion of dozen-egg, eggshell percentage, viability and moisture in the excreta of birds. Diets containing $0.281 \% \mathrm{Na}$, corresponding to a daily intake of $69.1 \mathrm{mg} \mathrm{Na} / \mathrm{hen}$, increased performance and egg quality of laying Japanese quail during the peak of production. However, a satisfactory performance, based on production of egg/ hen.day and commercial egg production can be obtained with birds fed diet containing 0,149\% de Na.
\end{abstract}

Key Words: Coturnix coturnix japonica, egg quality, egg production, salt

\section{Introdução}

A codorna tem sido considerada um produto alternativo na produção de carne e ovos. A pequena exigência de espaço, o baixo consumo de ração, o curto intervalo de geração, a maturidade sexual precoce e a persistência em elevada produção de ovos são características vantajosas em comparação a outras aves. Além disso, destacam-se a alta qualidade dos produtos finais obtidos na exploração comercial e o fácil manejo na criação destas aves.

Atualmente, a nutrição tem sido pesquisada visando constantes melhorias nos índices produtivos das aves e, em virtude do progresso genético aplicado a esta espécie, é necessário estabelecer e atualizar constantemente os níveis adequados de nutrientes da dieta. Entre os nutrientes mais estudados, os minerais participam de processos bioquímicos 
(por meio da ativação de sistemas enzimáticos) do processo de absorção e de transporte de nutrientes no organismo.

O Na é um cátion monovalente encontrado principalmente nos líquidos extracelulares. Realiza importantes funções metabólicas como o equilíbrio do volume hídrico e do $\mathrm{pH}$, a transmissão de impulsos nervosos e a manutenção das contrações musculares e cardíacas e participa na absorção de vitaminas hidrossolúveis, como riboflavina, tiamina e ácido ascórbico e água (Mc Dowell, 1992).

Em razão do envolvimento do Na em funções metabólicas essenciais, a deficiência deste mineral influencia negativamente o desempenho produtivo dos animais. Em galinhas poedeiras alimentadas com dietas com níveis de $\mathrm{Na}$ insuficientes, observam-se baixo consumo de ração, redução e rápida interrupção da postura, retardo no crescimento e comportamento de canibalismo (Kuchinski et al., 1997).

De acordo com Puls (1988), o nível crítico de deficiência estaria entre 0,012 e 0,05\% de $\mathrm{Na}$ na dieta de frangos de corte e de galinhas poedeiras. Segundo o NRC (1994), a recomendação de Na para codorna japonesa em fase de postura é de $0,15 \%$.

Sakamoto et al. (2004), objetivando determinar o melhor nível de suplementação de sal comum em dietas para codornas japonesas em fase de postura, utilizaram níveis de 0,0 ; 0,$15 ; 0,20 ; 0,25 ; 0,30 ; 0,35$ e $0,45 \%$, correspondentes a 0,04 ; 0,$10 ; 0,12 ; 0,14 ; 0,16 ; 0,18$ e $0,22 \%$ de $\mathrm{Na}$, respectivamente, e constataram que a dieta sem adição de sal ( $0,04 \%$ de $\mathrm{Na})$ comprometeu os resultados de desempenho e promoveu resposta inferior para todas as variáveis avaliadas, exceto a unidade Haugh, que se comportou de modo não-significativo nos níveis de Na estudados. Os autores concluíram que o nível de $0,15 \%$ de sal, equivalente a $0,10 \%$ de Na, seria o mais adequado para obtenção de melhor desempenho e qualidade externa dos ovos.

Ao estudarem níveis de Na em dietas $(0,11 ; 0,14 ; 0,17$; 0,20 e $0,23 \%$ ) para codornas japonesas durante o período de verão, Figueiredo et al. (2004) recomendaram o nível de $0,146 \%$ de Na como o mais adequado para a fase de postura. No entanto, o excesso de Na causa também problemas produtivos, pois, assim como o cloro, doses elevadas de Na resultam em aumentos na absorção de água em aves (Hooge, 1999).

O aumento do consumo de água, uma das principais conseqüências da ingestão de níveis excessivos de $\mathrm{Na}$, faz com que as aves produzam excretas mais úmidas e com maiores quantidades de $\mathrm{Na}$, reduzindo a taxa de filtração glomerular (Wideman et al., 1985).

Apesar da importância para a manutenção destas importantes funções vitais, o Na tem recebido pouca atenção dos nutricionistas em pesquisas com codornas em postura, em virtude do baixo custo e da disponibilidade. Segundo Murakami \& Furlan (2002), em decorrência da falta de informação da correta exigência de Na para codornas, os nutricionistas têm utilizado aproximadamente 0,25 a $0,30 \%$ de cloreto de sódio na ração, de modo a atender às necessidades da ave.

Objetivou-se nesta pesquisa estudar os efeitos de diferentes níveis de Na na dieta sobre o desempenho e a qualidade de ovos de codornas japonesas durante o pico de produção.

\section{Material e Métodos}

O experimento foi realizado no Setor de Avicultura do Departamento de Zootecnia da Universidade Federal de Viçosa, Minas Gerais, no período de 12 de setembro a 13 de novembro de 2005.

Foram utilizadas 300 codornas japonesas durante o pico de postura (69 a 132 dias de idade), com peso inicial médio de 168,0 g, aos 69 dias de idade, alojadas em baterias metálicas. Cada bateria foi composta de cinco gaiolas dispostas em andares, constituídas de arame galvanizado. Cada gaiola foi composta de três unidades experimentais, com dimensões de $96 \times 37 \times 16 \mathrm{~cm}$ (largura $\times$ profundidade $\times$ altura), cada uma com dez aves.

As gaiolas foram equipadas com comedouros e bebedouros do tipo calha, ambos colocados em toda a extensão da gaiola (o comedouro posicionado na parte frontal e o bebedouro na parte posterior), além de uma bandeja colocada sob a gaiola para recolhimento das excretas. Cada comedouro foi equipado com divisórias em madeira, coincidindo com a largura de cada unidade experimental.

A temperatura foi medida duas vezes ao dia (8 e 16h), por meio de termômetros de máxima e de mínima, mantidos na altura média das baterias e posicionados no centro da sala. A água e a ração foram fornecidas duas vezes por dia (8 e 16h). A coleta dos ovos foi realizada no horário da manhã, logo após o manejo.

A iluminação do ambiente foi feita por uma lâmpada incandescente de 100 watts controlada por um timer, com fornecimento de 17 horas de luz por dia.

O delineamento experimental utilizado foi o inteiramente casualizado, composto de cinco tratamentos (níveis de $\mathrm{Na}$ ), seis repetições e dez aves por unidade experimental.

As dietas experimentais foram formuladas à base de milho e farelo de soja, com base na composição dos ingredientes descrita por Rostagno et al. (2005), e foram isoenergéticas, isoprotéicas e isocálcicas, atendendo às exigências nutricionais preconizadas pelo NRC (1994), exceto para lisina e cálcio, cujos níveis foram calculados, 
Tabela 1 - Composições percentuais e químicas e valores nutricionais das dietas (\% MN)

Table 1 - Percentage and chemical compositions and nutritional values of the diets (\% as-fed)

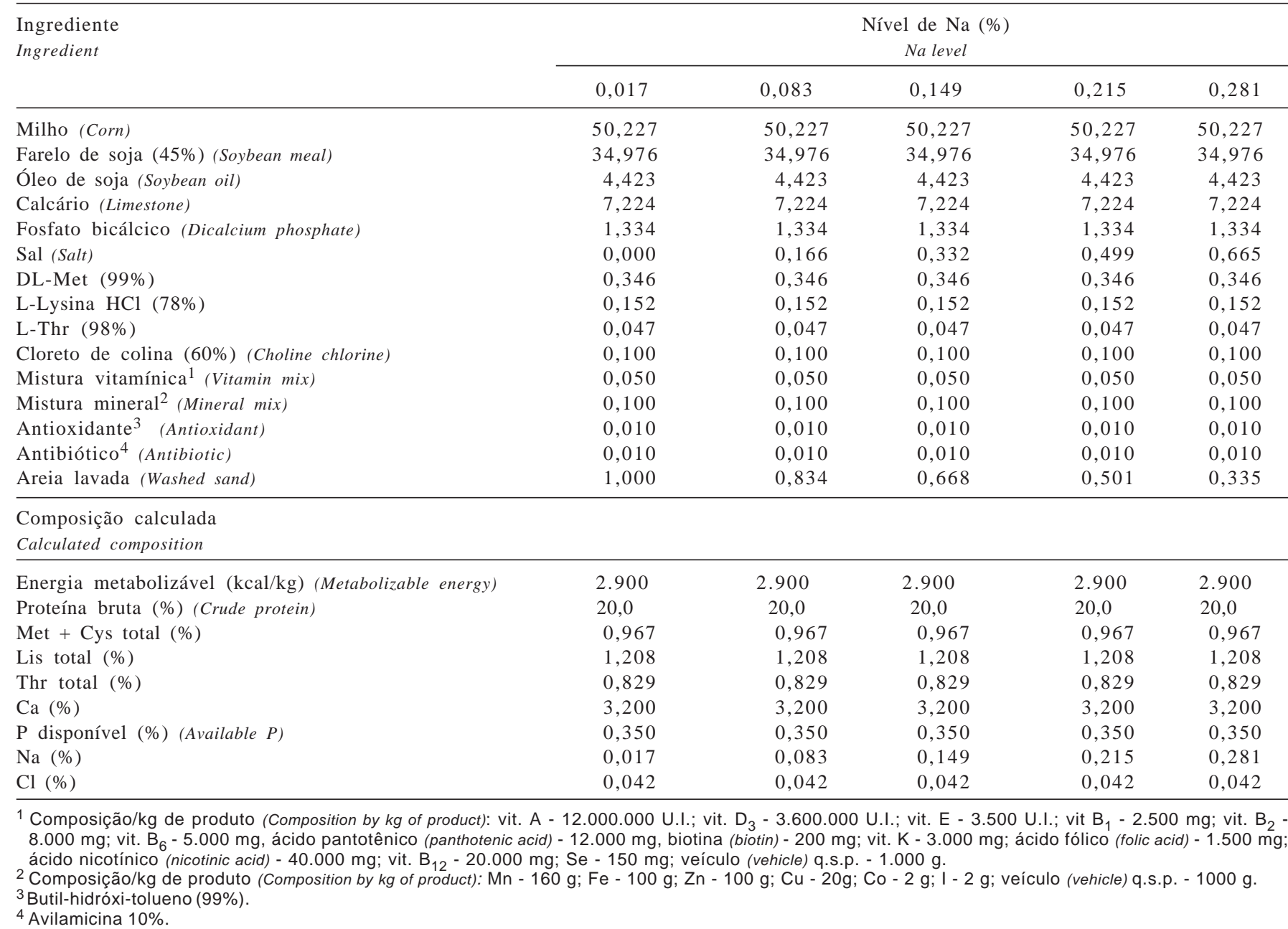

respectivamente, segundo recomendações de Pinto et al. (2003) e Pereira (2004). A relação dos aminoácidos:lisina foram de $80 \%$ para metionina + cistina e $65 \%$ para treonina.

Os níveis de Na das dietas foram obtidos pela substituição do inerte (areia) por cloreto de sódio (sal comum) e resultaram em 0,$083 ; 0,149 ; 0,215$ e $0,281 \%$, exceto para a dieta controle, com $0,017 \%$ de $\mathrm{Na}$, proveniente do milho e do farelo de soja, ou seja, sem adição de sal comum (Tabela 1).

No estudo do desempenho das aves, foram considerados a produção de ovos/ave/dia, a produção de ovos/ave alojada, a produção de ovos comercializáveis, o consumo de ração, a conversão alimentar (por massa e por dúzia de ovos), o peso médio dos ovos, a massa de ovos/ave/dia, os pesos corporais inicial e final e a viabilidade econômica da dieta. Para avaliação da qualidade dos ovos, foram analisadas as porcentagens de casca, de albúmen e de gema, a espessura de casca, a gravidade específica e a unidade Haugh. Além disso, foi calculada a umidade das excretas das aves.
Para recolhimento das excretas para posterior análise de matéria seca, foi colocada uma bandeja de chapa metálica galvanizada, encapada com plástico preto sob o piso das gaiolas. O teor de umidade foi obtido a partir da diferença de peso de amostras antes e depois de permanecerem em estufa de ventilação forçada, por três dias a $72^{\circ} \mathrm{C}$.

Os parâmetros avaliados foram submetidos à análise de variância pelo programa SAEG - Sistema para Análises Estatísticas e Genéticas (UFV, 1999). Os efeitos dos níveis de Na foram estimados pelos modelos de regressão linear e quadrática, conforme o melhor ajustamento obtido para cada variável, considerando o comportamento biológico da espécie.

\section{Resultados e Discussão}

As temperaturas mínima média e máxima média do ar foram de 22,5 e $26,6^{\circ} \mathrm{C}$, respectivamente, e a umidade relativa média do ar foi de $74 \%$. 
Tanto para as variáveis relacionadas ao desempenho quanto para aquelas relacionadas à qualidade dos ovos, a dieta controle, sem suplementação de sal comum $(0,017 \%$ de $\mathrm{Na}$ ), não pôde ser comparada às demais, uma vez que a postura das aves foi interrompida na primeira semana do período experimental e as aves alimentadas com esta dieta foram mantidas fora da produção durante todo o período experimental, o que prejudicou a avaliação de todas variáveis para esta dieta. Desse modo, a análise estatística foi feita comparando-se apenas os quatro maiores níveis de $\mathrm{Na}$ estudados.

Verificou-se efeito linear positivo dos níveis de Na na produção de ovos/ave/dia $(\mathrm{P}<0,01)$, na produção de ovos por ave alojada $(\mathrm{P}<0,05)$, na produção de ovos comercializáveis $(\mathrm{P}<0,01)$, na conversão alimentar por massa de ovos $(\mathrm{P}<0,05)$, nas porcentagens de albúmem e de gema no ovo $(\mathrm{P}<0,01)$ e na gravidade específica dos ovos $(\mathrm{P}<0,01)$. Houve comportamento quadrático para o consumo de ração $(\mathrm{P}<0,01)$, o peso médio do ovo $(\mathrm{P}<0,01)$, a massa de ovo/ave/dia $(\mathrm{P}<0,05)$, o peso corporal final $(\mathrm{P}<0,01)$, a espessura de casca $(\mathrm{P}<0,05)$ e a unidade Haugh do ovo $(\mathrm{P}<0,01)$. Não foram encontrados
$(\mathrm{P}>0,05)$ efeitos dos níveis de Na sobre a conversão alimentar por dúzia de ovos, o peso corporal inicial, a porcentagem de casca no ovo, a viabilidade e a umidade na excreta das aves.

O consumo diário de ração aumentou significativamente nas aves alimentadas com a dieta contendo até $0,22 \%$ de Na e diminuiu naquelas alimentadas com dieta contendo níveis mais elevados de $\mathrm{Na}$ (Tabelas 2 e 3). Faria et al. (2000) observaram resultados não-significativos para consumo de ração de galinhas poedeiras alimentadas com dietas contendo diferentes níveis de $\mathrm{Na}(0,16 ; 0,20$ e $0,24 \%)$ e fósforo $(0,35 ; 0,45$ e $0,55 \%)$. Do mesmo modo, Rodrigues et al. (2004), ao avaliarem o efeito de níveis de $\mathrm{Na}(0,15 ; 0,25$ e $0,35 \%$ ) em dietas para galinhas poedeiras no segundo ciclo de postura, não obtiveram diferenças entre as dietas. Entretanto, Fassani et al. (2002) observaram que níveis de 0,05 a $0,25 \%$ de Na adicionados à dieta de galinhas poedeiras no segundo ciclo de produção promoveram efeito quadrático, com nível ótimo de $0,188 \%$ de Na. No caso de codornas japonesas, os resultados diferem também dos relatados por Figueiredo et al. (2004), que não observaram diferenças no consumo de ração entre os níveis de $\mathrm{Na}$ estudados.

Tabela 2 - Consumo de ração (CR), produção de ovos/ave/dia (POAD), produção de ovos ave alojada (POAA), produção de ovos comercializáveis (POC), peso médio do ovo (PMO), massa de ovo (MO), conversão alimentar por dúzia de ovos (CADZ), conversão alimentar por massa de ovos (CAMO), peso corporal inicial (PI), peso corporal final (PF), viabilidade (VIA), porcentagens de casca (PC), de albúmem (PA) e de gema (PG), espessura de casca (EC), gravidade específica (GE), unidade Haugh (UH) do ovo e umidade da excreta (UE) em codorna japonesa alimentada com dietas com diferentes níveis de $\mathrm{Na}$

Table 2 - Feed intake (FI), egg/hen/day production (EHDP), egg/hen/housed production (EHHP), commercial egg (CEP), egg weight (EW), egg mass (EM), feed conversion by dozen of egg (FCDZ), feed conversion by egg mass (FCEM), initial body weight (IW), final body weight (FW), viability (VIA) shell percentage (SP), albumen percentage (AP), yolk percentage (YP), shell thickness (ST), specific gravity (SG), egg Haugh unit (HU) and feces humidity (FU) of Japanese quail fed diets with different Na levels

\begin{tabular}{|c|c|c|c|c|c|}
\hline \multirow[t]{2}{*}{ Item } & \multicolumn{4}{|c|}{$\begin{array}{c}\text { Nível de Na (\%) } \\
\text { Na level }\end{array}$} & \multirow[t]{2}{*}{ CV $(\%)$} \\
\hline & 0,083 & 0,149 & 0,215 & 0,281 & \\
\hline $\operatorname{POAD}(\%)^{2}(E H D P)$ & 89,61 & 96,26 & 92,82 & 96,32 & 2,70 \\
\hline POAA $(\%)^{3}$ (EHHP) & 87,22 & 95,24 & 89,87 & 96,32 & 4,37 \\
\hline POC $(\%)^{2}(C E P)$ & 86,96 & 94,96 & 90,45 & 94,89 & 3,66 \\
\hline CADZ $(\mathrm{kg} / \mathrm{dz})(F C D Z)$ & 0,314 & 0,313 & 0,313 & 0,305 & 3,61 \\
\hline CAMO $(\mathrm{kg} / \mathrm{kg})^{3}$ (FCEM) & 2,198 & 2,121 & 2,117 & 2,074 & 3,28 \\
\hline PI (g) (IW) & 170,0 & 169,0 & 167,6 & 167,3 & 2,76 \\
\hline $\operatorname{PF}(g)^{5}(F W)$ & 176,2 & 186,5 & 186,8 & 182,6 & 3,59 \\
\hline VIA (\%) (VIA) & 95,00 & 98,33 & 96,67 & 100,00 & 5,75 \\
\hline PC (\%) (SP) & 8,02 & 8,16 & 8,10 & 8,06 & 3,00 \\
\hline $\mathrm{UH}^{5}(\mathrm{HU})$ & 89,66 & 88,63 & 89,06 & 89,27 & 1,35 \\
\hline UE (\%) (FU) & 74,98 & 75,35 & 74,83 & 76,21 & 2,55 \\
\hline
\end{tabular}

${ }^{1}$ Coeficiente de variação (Coefficient of variation).

2 Efeito linear $(P<0,01)$ (Linear effect).

3 Efeito linear $(P<0,05)$ (Linear effect).

4 Efeito quadrático $(P<0,01)$ (Quadratic effect).

5 Efeito quadrático $(\mathrm{P}<0,05)$ (Quadratic effect). 
Tabela 3 - Equações de regressão das variáveis estudadas

Table 3 - Regression equations of the studied variables

\begin{tabular}{|c|c|c|}
\hline Item & $\begin{array}{c}\text { Equação de regressão } \\
\text { Regression equation }\end{array}$ & $\mathrm{R}^{2}$ \\
\hline Consumo de ração (Feed intake) & $\hat{Y}=20,727+35,072-78,856 X^{2}$ & 0,50 \\
\hline Produção de ovos/ave alojada (Egg production/housed hen) & $\hat{Y}=86,118+33,205 X$ & 0,43 \\
\hline Produção de ovos comercializáveis (Commercial egg production) & $\hat{\mathrm{Y}}=86,503+29,194 \mathrm{X}$ & 0,42 \\
\hline Peso médio do ovo (Egg weight) & $\hat{Y}=11,143+11,446 X-26,844 X^{2}$ & 0,95 \\
\hline Conversão alimentar por massa de ovo (Feed conversion per egg mass) & $\hat{\mathrm{Y}}=2,2311-0,5694 \mathrm{X}$ & 0,88 \\
\hline Peso corporal final (Final body weight) & $\hat{Y}=154,69+331,60 X-829,65 X^{2}$ & 0,98 \\
\hline Percentagem de albúmem (Albumen percentage) & $\hat{\mathrm{Y}}=62,944+3,9446 \mathrm{X}$ & 0,88 \\
\hline Percentagem de gema (Yolk percentage) & $\hat{\mathrm{Y}}=29,8902-4,0773 \mathrm{X}$ & 0,92 \\
\hline Espessura de casca (Shell thickness) & $\hat{Y}=17,525+29,611 X-72,626 X^{2}$ & 0,99 \\
\hline
\end{tabular}

A produção de ovos melhorou significativamente com o aumento dos níveis de Na na ração (Tabela 3). No entanto, em valores absolutos, a produção de ovos/ave/dia foi praticamente a mesma $(96,3 \%)$ nas aves alimentadas com ração contendo 0,149 e 0,281\% de Na (Tabela 2). O mesmo comportamento foi observado para a produção de ovos comercializáveis, que foi idêntica (94, 9\%) entre as dietas. Nesse caso, pode-se inferir que dieta contendo $0,149 \%$ de $\mathrm{Na}$ foi suficiente para obtenção de melhores resultados para a produção de ovos. Comportamentos diferentes são relatados na literatura em estudos para avaliação da exigência de Na para codornas. Figueiredo et al. (2004) obtiveram efeito quadrático, com nível ótimo de Na em 0,148\%, sobre a produção de ovos quando avaliaram rações contendo níveis de 0,11 a 0,23\% desse mineral. Ao contrário, Sakamoto et al. (2004), utilizando dietas com níveis de 0,04 a 0,22\% de $\mathrm{Na}$ para codornas japonesas, não observaram diferenças entre a produção de ovos obtida com a dieta contendo 0,10 a $0,22 \%$ de $\mathrm{Na}$, no entanto, a dieta sem adição de sal (controle) continha $0,04 \%$ de $\mathrm{Na}$, ou seja, o dobro da quantidade encontrada na dieta sem inclusão de sal deste trabalho (0,017\% de Na).

O peso médio do ovo aumentou até o nível de $0,213 \%$ de Na (Tabela 3), enquanto a massa diária de ovo/ave foi maximizada com o nível de $0,246 \%$ de Na na dieta, em razão do comportamento quadrático verificado para ambos os parâmetros. O resultado encontrado para o peso do ovo difere do obtido por Figueiredo et al. (2004), que não observaram diferenças ao fornecerem dietas suplementadas com sal para codornas japonesas no período de verão. Diferem também dos descritos por Rodrigues et al. (2004), que não encontraram efeito significativo dos níveis de $\mathrm{Na}$ nas dietas sobre essas variáveis em galinhas poedeiras comerciais no segundo ciclo de postura. Sakamoto et al. (2004), ao determinarem o melhor nível de sal para codornas japonesas, não observaram diferenças entre as médias das dietas suplementadas com sal comum ( 0,10 a $0,22 \%$ de $\mathrm{Na}$ ).

As dietas não diferiram entre si $(\mathrm{P}>0,05)$ quanto à conversão alimentar por dúzia de ovos (Tabela 2), o que está de acordo com o resultado encontrado por Murakami et al. (2003), que não encontraram diferenças na conversão alimentar por dúzia de ovos entre os níveis de Na estudados no primeiro e segundo ciclos de produção de galinhas poedeiras comerciais. No entanto, a conversão alimentar por massa de ovos comprovou efeito linear decrescente conforme aumentaram os níveis de Na das dietas, indicando melhora significativa em aves alimentadas com ração contendo 0,218\% de Na. Essa melhora da conversão pode ser atribuída à redução do consumo de ração, ao aumento na produção de ovos e à pequena redução no peso dos ovos das aves alimentadas com ração contendo o maior nível de $\mathrm{Na}$ (Tabelas 2 e 3). Esse resultado contraria os descritos por Pizzolante et al. (2005), que verificaram os piores resultados para conversão alimentar por massa de ovos em codornas alimentadas com ração contendo $0,22 \%$ de $\mathrm{Na}$, ao testarem níveis de 0,10 a $0,22 \%$ desse mineral em rações para codornas japonesas em final de produção (54 semanas de idade). Resultados diferentes, com efeito quadrático, foram verificados por Figueiredo et al. (2004), que concluíram como nível ideal o de $0,146 \%$ de Na para obtenção de melhor conversão por massa em codornas japonesas no período de verão. Fassani et al. (2002) observaram também resposta quadrática (nível ótimo de $0,182 \% \mathrm{Na}$ ) ao avaliarem o efeito do Na na dieta de galinhas poedeiras. Murakami et al. (2003) 
e Rodrigues et al. (2004) obtiveram resultados não-significativos para conversão alimentar em galinhas poedeiras no segundo ciclo de postura alimentadas com dietas suplementadas com níveis crescentes de Na.

O peso corporal das aves no final do experimento apresentou comportamento quadrático, com aumento crescente nas aves alimentadas com a ração contendo até 0,199\% de Na (Tabela 3). A partir deste nível houve redução de aproximadamente 2,3\% no peso para aves alimentadas com a ração contendo o maior nível de $\mathrm{Na}(0,281 \%)$, o que não comprometeu o desempenho das aves, avaliado pela produção e conversão alimentar (Tabela 2). Resultado semelhante foi encontrado por Fassani et al. (2002), que verificaram efeito quadrático dos níveis de Na sobre o peso corporal de galinhas poedeiras ao final do experimento.

As porcentagens de albúmen e de gema apresentaram comportamento linear crescente e decrescente, respectivamente, com o aumento dos níveis de Na na dieta (Tabela 3). No entanto, efeito não-significativo foi observado para porcentagem de casca, que apresentou comportamento estável mesmo nos ovos provenientes de aves alimentadas com ração contendo os maiores níveis de Na (Tabela 2). Esse resultado concorda parcialmente com o observado por Sakamoto et al. (2004), que, ao avaliarem a porcentagem de casca de ovos de codorna, verificaram diferenças entre as médias do nível de 0,04 (sem adição de sal) e 0,10\% de Na, porém, entre os níveis 0,10 a $0,22 \%$ de $\mathrm{Na}$, as diferenças não foram detectadas.

Avaliando a qualidade da casca do ovo pela espessura, verificou-se comportamento quadrático dos níveis de $\mathrm{Na}$, indicando maximização da casca em ovos de codornas alimentadas com ração contendo $0,204 \%$ de Na (Tabela 3). A gravidade específica do ovo, no entanto, um parâmetro que determina indiretamente a qualidade da casca, elevou significativamente com o aumento dos níveis de Na na dieta das aves (Tabela 3), o que confirma a melhora linear na produção de ovos comercializáveis (sem problemas de casca) com o aumento dos níveis de Na na dieta. Entretanto, Faria et al. (2000), avaliando o efeito dos níveis de Na e fósforo em dietas para galinhas poedeiras comerciais, e Sakamoto et al. (2004) e Pizzolante et al. (2005), em estudos com codornas japonesas, não observaram efeito dos níveis de Na na dieta sobre a espessura da casca dos ovos. Em pesquisas com codornas japonesas, Figueiredo et al. (2004) e Pizzolante (2005) verificaram efeito não-significativo do nível de Na sobre a gravidade específica do ovo.

A unidade Haugh do ovo apresentou comportamento quadrático (Tabela 3), indicando menores valores para ovos de codornas alimentadas com ração contendo $0,19 \%$ de Na, o que reforça a melhora da qualidade de ovo proveniente de aves alimentadas com ração contendo níveis mais elevados de Na (Tabela 2). Outras pesquisas relatam efeito nãosignificativo para a unidade Haugh em ovos de codornas japonesas e galinhas poedeiras alimentadas com ração contendo níveis diferenciados de Na (Figueiredo et al., 2004; Sakamoto et al., 2004; Rodrigues et al., 2004).

A umidade das excretas não foi afetada pela suplementação de Na nas dietas $(\mathrm{P}>0,05)$. As aves alimentadas com a dieta sem suplementação de sal comum $(0,017 \% \mathrm{Na})$ permaneceram todo o período experimental em aspecto de desidratação e com baixo consumo de ração, ou seja, é possível que a falta do íon Na tenha dificultado a absorção de água e de nutrientes pelo organismo, resultando em pausa na produção durante todo o período experimental. Segundo Pizzolante et al. (2005), as variações no equilíbrio ácido-base e na pressão osmótica podem afetar o crescimento, o consumo de ração e a qualidade de casca dos ovos de codornas. Além disso, o Na, o cloro e o potássio são elementos importantes para manter esse equilíbrio eletrolítico.

Sugere-se que outros trabalhos para avaliação de níveis de Na superiores aos avaliados nesta pesquisa sejam realizados com codornas em postura, uma vez que melhores resultados de desempenho e de qualidade de ovos foram verificados em aves que receberam ração contendo o maior nível de $\mathrm{Na}(0,281 \%)$, obtido com a inclusão de $0,665 \%$ de sal comum na ração à base de milho e farelo de soja (Tabela 1).

\section{Conclusões}

A suplementação de $0,281 \%$ de Na na dieta, correspondente a um consumo diário de 69,1 mg de Na/ave, melhora o desempenho e a qualidade do ovo de codornas japonesas durante o pico de produção. No entanto, desempenho satisfatório, calculado com base na produção de ovos/ave/ dia e de ovos comercializáveis, pode ser obtido com o fornecimento de rações contendo 0,149\% de Na.

\section{Literatura Citada}

FARIA, D.E.; JUNQUEIRA, O.M.; SAKOMURA, N.K. et al. Efeito de diferentes níveis de sódio e fósforo sobre o desempenho e a qualidade da casca dos ovos de poedeiras comerciais. Revista Brasileira de Zootecnia, v.29, n.2, p.458-466, 2000.

FASSANI, E.J.; BERTECHINI, A.G.; BRITO, J.A.G. et al. Utilização de diferentes níveis de suplementação de sódio para poedeiras comerciais no segundo ciclo de produção. Revista Brasileira de Ciência Avícola, v.4, n.3, p.235-241, 2002.

FIGUEIREDO, G.O.; KATO, R.K.; BERTECHINI, A.G. et al. Níveis de sódio para codornas japonesas (Coturnix coturnix japonica) no verão. In: SIMPÓSIO INTERNACIONAL, 2., CONGRESSO BRASILEIRO DE COTURNICULTURA, 1., 2004, Lavras. Anais... Lavras: Universidade Federal de Lavras, 2004. p.216. 
HOOGE, D.M. A importância dos eletrólitos. Avicultura Industrial, n.1068, p.20-26, 1999.

KUCHINSKI, K.K.; HARMS, R.H. Re-evaluation of the sodium of the commercial laying hen. Official Journal of the poultry Science Association, v.76, n.1, p.59, 1997 (suppl.).

NATIONAL RESEARCH COUNCIL - NRC. Nutrient requirements of poultry. 9.ed. Washington, D.C.: National Academy of Sciences, 1994. 155p.

MC DOWELL, L.R. Minerals in animal and human nutrition. London: Academic Press, 1992. 524p.

MURAKAMI, A.E.; FURLAN, A.C. Pesquisas na nutrição e alimentação de codornas em postura no Brasil. In: SIMPÓSIO INTERNACIONAL DE COTURNICULTURA, 1., 2002, Lavras. Anais... Lavras: Universidade Federal de Lavras, 2002. p.113-120.

MURAKAMI, A.E.; FIGUEIREDO, D.F.; PERUZZI, A.Z. et al. Níveis de sódio para poedeiras comerciais no primeiro e segundo ciclos de produção. Revista Brasileira de Zootecnia, v.32, n.6, p.1674-1680, 2003 (supl. 1).

PEREIRA, C.A. Exigência nutricional de cálcio para codornas japonesas durante o pico de postura. Viçosa, MG: Universidade Federal de Viçosa, 2004. 60p. Dissertação (Mestrado em Zootecnia) - Universidade Federal de Viçosa, 2004.

PINTO, R.; FERREIRA, A.S.; DONZELE, J.L. et al. Exigência de lisina para codornas japonesas em postura. Revista brasileira de Zootecnia, v.32, n.5, p.1182-1189, 2003.

PIZZOLANTE, C.C.; SALDANHA, E.S.P.B.; GARCIA, E.A. et al. Níveis de sal comum em rações de codornas japonesas (Coturnix japonica) em final de produção. In: CONGRESSO DE PRODUÇÃO, CONSUMO DE OVOS E COMERCIALIZAÇÃO DE OVOS - APA, 3., 2005, São Paulo. Anais... São Paulo: 2005. p.89.
PULS, R. Mineral levels in animal health. Clearbook: Sherpa International, 1988. 438p.

RODRIGUES, E.A.; JUNQUEIRA, O.M.; VALÉRIO, M. et al. Níveis de sódio em rações de poedeiras comerciais no segundo ciclo de postura. Revista Brasileira de Zootecnia, v.33, n.2, p.391396, 2004.

ROSTAGNO, H.S.; ALBINO, L.F.T.; DONZELE, J.L. et al. Composição de alimentos e exigências nutricionais (Tabelas brasileiras para aves e suínos). 2.ed. Viçosa, MG: Universidade Federal de Viçosa, 2005. 186p.

SAKAMOTO, M.I.; MURAKAMI, A.E.; SUZUKI, E.T. et al. Determinação do melhor nível de sal comum para codornas japonesas em postura (Coturnix coturnix japonica). In: SIMPÓSIO INTERNACIONAL, 2., CONGRESSO BRASILEIRO DE COTURniCUlturA, 1., 2004, Lavras. Anais... Lavras: Universidade Federal de Lavras, 2004. p.217.

WIDEMAN, R.F.; BUSS, E.G. Arterial blood gas, pH, and bicarbonate values in laying hens for thin eggsshell production. Poultry Science, v.64, p.1015-1019, 1985.
Recebido: 20/11/2006 Aprovado: $16 / 4 / 2007$ 